\title{
Cardiac-Motion Compensated MR Imaging and Strain Analysis of Ventricular Trabeculae
}

\author{
Andrew W. Dowsey ${ }^{1}$, Jennifer Keegan ${ }^{2}$, and Guang-Zhong Yang ${ }^{1}$ \\ ${ }^{1}$ Institute of Biomedical Engineering, Imperial College London, SW7 2AZ, UK \\ \{a.w.dowsey, g.z.yang\} @imperial.ac.uk \\ ${ }^{2}$ Cardiovascular Magnetic Resonance Unit, National Heart and Lung Institute, \\ Imperial College London, Royal Brompton and Harefield NHS Trust, SW3 6NP, UK \\ j.keegan@rbht.nhs.uk
}

\begin{abstract}
In conventional CMR, bulk cardiac motion causes target structures to move in and out of the static acquisition plane. Due to the partial volume effect, accurate localisation of subtle features through the cardiac cycle, such as the trabeculae and papillary muscles, is difficult. This problem is exacerbated by the short acquisition window necessary to avoid motion blur and ghosting, especially during early systole. This paper presents an adaptive imaging approach with COMB multi-tag tracking that follows true 3D motion of the myocardium so that the same tissue slice is imaged throughout the cine acquisition. The technique is demonstrated with motion-compensated multi-slice imaging of ventricles, which allows for tracked visualisation and analysis of the trabeculae and papillary muscles for the first time. This enables novel in-vivo measurement of circumferential and radial strain for trabeculation and papillary muscle contractility. These statistics will facilitate the evaluation of diseases such as mitral valve insufficiency and ischemic heart disease. The adaptive imaging technique will also have significant implications for CMR in general, including motioncompensated quantification of myocardial perfusion and blood flow, and motion-correction of sequences with long acquisition windows.
\end{abstract}

\section{Introduction}

\begin{abstract}
Abnormality in the trabeculae carnae, a network of predominantly longitudinal muscular ridges that line the left and right ventricles, has been identified as a significant factor in a number of serious cardiomyopathies. Extensive non-compacted myocardium (isolated ventricular non-compaction) can lead to arrhythmia, thromboembolism and cardiac failure [1]. Similarly, the pathogenic replacement of trabeculae with fatty or fibrous tissue in arrythmogenic ventricular dysplasia can cause an electrical instability and is the primary cause of sudden cardiac arrest in young adults [2]. Amongst the trabeculae, at the base of both ventricles, reside the papillary muscles. The left ventricle contains two groups of papillary muscle that attach to the mitral valve leaflets through the chordae tendinae. They contract a fraction earlier in the cardiac cycle to ensure the mitral valve stays closed during systole. However, in patients with ischemic heart disease, displacement of the papillary muscles causes mitral insufficiency, both directly through ischemic dysfunction, and indirectly through dilation of
\end{abstract}


the left ventricle [3]. In basic haemodynamics, it is thought that the trabeculations reduce the turbulence of blood during systole. However, since detailed in-vivo measurement of trabecula and papillary muscle motion has not been possible due to their small structure and rapid systolic motion [4], the hypothesis remains unproved. Indeed, with advances in multi-detector CT, it was only discovered recently that the papillary muscles attach to the trabeculations rather than directly to the heart wall [5]. CT is now in common use for morphologic assessment of trabeculae in isolated ventricular non-compaction and arrythmogenic ventricular dysplasia. However, the required radiation dose involved limits its practical adoption. Furthermore, the temporal resolution is insufficient for accurate quantification of morphology and motion during the whole cardiac cycle.

Although recent developments in cardiovascular MR overcome many of these issues, cyclic through-plane motion causes structures to move in and out of the imaging plane, thus greatly affecting the depiction of trabecular morphology. To avoid this problem, it is necessary to adaptively track bulk movement of the heart during data acquisition so that the same structures remain localised throughout the cycle. The purpose of this paper is to present a prospective imaging scheme that tracks ventricular motion in real-time through a single breath-hold COMB multi-tag pre-scan. Our previous manually-seeded single tag tracking method [6] is extended through an objective and automatic real-time 4D multi-tag approach. This is performed whilst the subject remains in the scanner for subsequent motion-compensated multi-slice acquisition of the whole ventricle. Each $k$-space sample is corrected individually to ensure optimal motion compensation. Strain analysis of the trabeculae and papillary muscles was then quantified for the first time. Since traditional techniques such as MR tagging are too coarse to capture the fine detail and complex motion of the trabeculations [7], in this paper a method based on novel mass-invariant and bias-corrected free-form image registration of cylindrical and radial projections is presented.

\section{Method}

\subsection{Adaptive Imaging with COMB Multi-tagging}

The tagging technique implemented is an extension of that used by Kozerke et al. [8]. A cine gradient echo echo-planar sequence with a labelling pre-pulse (selective and non-selective $90^{\circ}$ pulse pair) was implemented on a Siemens Avanto 1.5 Tesla scanner. With this method, the labelled images are generated by the complex subtraction of two datasets, with the phase of the selective component of the labelling pulse pair being reversed between the two. Interleaved horizontal (HLA) and vertical (VLA) long axis image planes were acquired during a single 18 cardiac cycle breath-hold, with labelling performed in the orthogonal short-axis plane. In this study, the selective $90^{\circ}$ pre-pulse is a COMB radiofrequency pulse, which enables the simultaneous labelling of 4 parallel short axis planes (plane thickness $=7 \mathrm{~mm}$, plane separation $=$ $20 \mathrm{~mm}$ ) centred in the middle of the left ventricle, as shown in Fig. 1(c-d). The parameters for the imaging sequence were as follows: slice thickness $=15 \mathrm{~mm}$, field of view $=400 \times 260 \mathrm{~mm}$, matrix size $=128 \times 71$, echo-planar readout factor $=8$ and temporal resolution (per interleaved cine pair) $=55 \mathrm{~ms}$. 

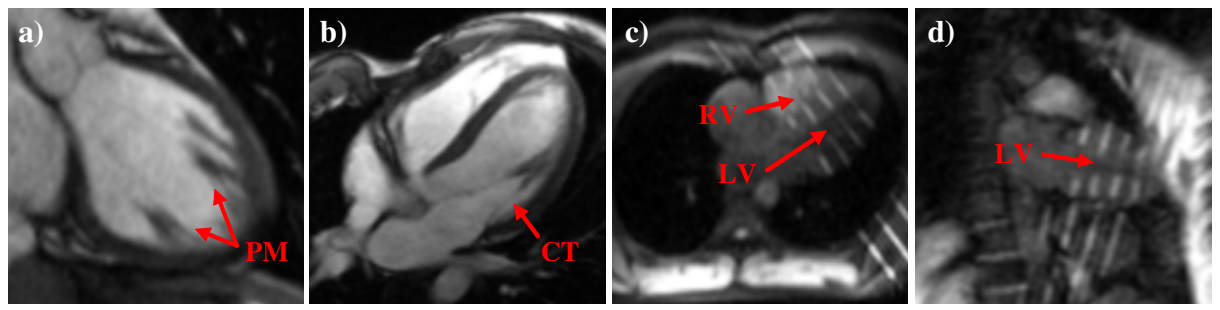

Fig. 1. (a) Mid-diastolic oblique view through the left ventricular papillary muscles (PM). (b) Perpendicular oblique view through lateral muscle showing connection of mitral valve to heads by chordae tendineae (CT). (c) Horizontal Long Axis (HLA) view showing 4 short-axis COMB tags through the left (LV) and right (RV) ventricles. (d) Perpendicular Vertical Long Axis (VLA) view showing the COMB tags placed through the left ventricle. (c) and (d) illustrate a systolic HLA/VLA pair in a single breath-hold COMB acquisition before complex subtraction.

In previous work, motion of a single COMB tag was tracked on HLA and VLA views using multi-resolution image registration with a hierarchical piece-wise bilinear transformation and BFGS optimization of the cross-correlation [6]. A single manual delineation on each of the two views was automatically propagated to the rest of the cardiac cycle, with the orthogonal distance regression plane calculated from the result.

To extend the tracking to multiple tagging of the whole ventricle, the regression plane was computed for each tag separately and Catmull-Rom interpolation used to derive the slice position and orientation for any slice offset between them. To delineate the tag objectively, a multi-resolution search was initiated along the tracked tag centerline in the set of acquired images, outwards from the tag centre, with the known tag location immediately after the ECG R-wave used as reference geometry. Gradient descent was used to minimize the sum of squared differences between the maximum observed intensity and the intensity of the tracked point through the cardiac cycle.

The result of the tracking is a list of the estimated sagittal, coronal and transverse components of the position and normal vectors of the COMB tags at all points in the cardiac cycle. A shared-phase cine TrueFISP sequence was adapted to read this output and to modify the orientation and offset (both through-plane and in-plane) for each $k$ space acquisition accordingly. Moving-slice breath-hold cine acquisitions were performed with a $300 \times 300 \mathrm{~mm}$ field of view (spatial resolution: $1.2 \times 1.2 \mathrm{~mm}$ ) and a $6 \mathrm{~mm}$ slice thickness. Twenty $k$-space samples (taking 60ms) were acquired per cardiac phase, with view-sharing enabling the reconstruction of data at $30 \mathrm{~ms}$ intervals.

\subsection{In-vivo Trabecular Strain Analysis}

With slice-tracked coverage of the heart, it is now possible to visualize and quantify trabeculae and papillary muscle motion over the whole cardiac cycle. Furthermore, when the slices are arranged and visualised as a 4D volume, bulk cardiac motion is frozen. However, for accurate motion quantification in this coordinate space, it is essential to ensure mass conservation with the world coordinate space. Isotrophic mass-conserved volumes were derived by taking each longitudinal stack of voxels and deriving the change in sampling rate in world coordinates through the stack: 


$$
I=\left|\operatorname{det}\left(\beta_{x}^{\prime}, \beta_{y}^{\prime}, \beta_{z}^{\prime}\right)\right| \beta_{i}
$$

where $\left(\beta_{\mathbf{x}}, \beta_{\mathbf{y}}, \beta_{\mathbf{z}}\right)$ is a uniform cubic $\mathrm{B}$-spline curve fitted [9] to the world coordinates of each longitudinal stack of voxels, and $\beta_{\mathrm{i}}$ is a uniform cubic B-spline curve fitted to the intensity values. $I(z)$ therefore provides cubic B-spline interpolation weighted by the change in sampling rate at point $z$. The epicardial border and papillary muscles were then delineated with piecewise Catmull-Rom contours and the trabeculae segmented from the blood pool using a Gaussian Mixture Model (GMM) with expectation-maximization. Each output slice was then transformed into a one dimensional vector by summation of the densities along in-plane radial lines projected from the centre of the ventricle, so that each volume became a cylindrical manifold of radial density parameterised by in-plane angle and longitudinal slice location.

Circumferential motion could then be derived between volumes by performing free-form image registration [10] on the cylindrical manifolds generated in the previous step. To provide a realistic $\mathrm{C} 2$ continuous deformation field, a Haar multi-resolution pyramid and hierarchical uniform cubic B-spline tensor-product transformation model, periodic in the circumferential dimension, was employed, as previously demonstrated for smooth-wall myocardial strain analysis by MR tagging [7]. To avoid convergence to local optima, global motion was first derived using a single B-spline patch on heavily sub-sampled images (16×16 pixels). The B-spline patch was then iteratively sub-divided 5 times and the resolution increased so that motion was derived at different scales up to $16 \times 16 \mathrm{~B}$-spline patches on $512 \times 512$ pixel images.

Surface receiver coil signal intensity variations contaminate the MR images with a multiplicative bias-field. The bias-field affects the computation of the GMM, and therefore myocardial mass. An additive bias-field can also be observed due to errors in epicardial delineation regionally over or under-estimating radial density. Since the bias-fields are regionally continuous, in this study they are modelled as hierarchal tensor-product B-spline surfaces, which are optimized concurrently with the transformation model using the same multi-resolution schedule. The complete sampling function for the source image is therefore:

$$
W_{s, \mathbf{C}}=\left|\operatorname{det}\left(\beta_{\mathbf{\theta}}^{\prime}, \beta_{\mathbf{Z}}^{\prime}\right)\right| \cdot\left(I_{s}\left(\beta_{\boldsymbol{\theta}}, \beta_{\mathbf{Z}}\right)+\beta_{\mathbf{V}}\right) \cdot e^{\beta_{\mathrm{U}}}
$$

where image $I_{s}$ is under geometric transformation $\left(\beta_{\boldsymbol{\theta}}, \beta_{\mathrm{Z}}\right)$, multiplicative bias-field correction $\beta_{\mathbf{U}}$, and additive bias-field correction $\beta_{\mathbf{V}}$. Tissue incompressibility is preserved by weighting the deformed density projection by its change in volume $\left|\operatorname{det}\left(\beta^{\prime}{ }_{\boldsymbol{\theta}}, \beta_{\mathbf{Z}}{ }_{\mathbf{Z}}\right)\right|$. The total set of parameters for optimization is therefore $\mathbf{C}=\{\boldsymbol{\theta}, \mathbf{Z}, \mathbf{U}, \mathbf{V}\}$. Since we deal with intensity correspondence between images explicitly with bias-field modeling, the similarity measure that drives the registration can be the sum of squared differences, defined as: $\operatorname{sim}\left(W_{s, \mathrm{C}}, I_{r}\right)=\sum_{(x, y) \in I_{r}}\left(W_{s, \mathrm{C}}(x, y)-I_{r}(x, y)\right)^{2}$. For fast convergence with the quasi-Newtonian limited-memory BFGS optimizer [11], the first partial derivatives of the similarity measure with respect to the transformation parameters were derived in closed form, where each pixel $\mathbf{p}$ in the reference image $I_{r}$ is compared to its corresponding pixel in the deformed sample image $W_{s, \mathbf{C}}$ : 


$$
\frac{\partial}{\partial \mathbf{C}} \operatorname{sim}\left(W_{s, C}, I_{r}\right)=2 \sum_{\mathbf{p} \in I_{r}}\left[\left(W_{s, \mathbf{C}}(\mathbf{p})-I_{r}(\mathbf{p})\right) \cdot \frac{\partial W_{s, \mathbf{C}}(\mathbf{p})}{\partial \mathbf{C}}\right]
$$

Relative circumferential strain is then computed as the partial derivatives of $\beta_{\theta}$ in the derived transformation mesh.

Similarly, relative radial strain was computed by analysis of circumferential density projections, where density along in-plane concentric circles was summed to generate a manifold parameterised by in-plane radius and longitudinal slice location.

\section{Results}

Fig. 2 illustrates the effect of the adaptive slice-tracking in localization of the trabeculations. Motion-tracked left ventricles were acquired for 5 subjects and the right ventricle for one subject (ages 23 to 28, 1 female, 4 male), with an average study time of 50 minutes. 17 to 25 motion-tracked slices, spaced initially at $5 \mathrm{~mm}$ intervals, were acquired for each dataset depending on ventricle length, resulting in full coverage.

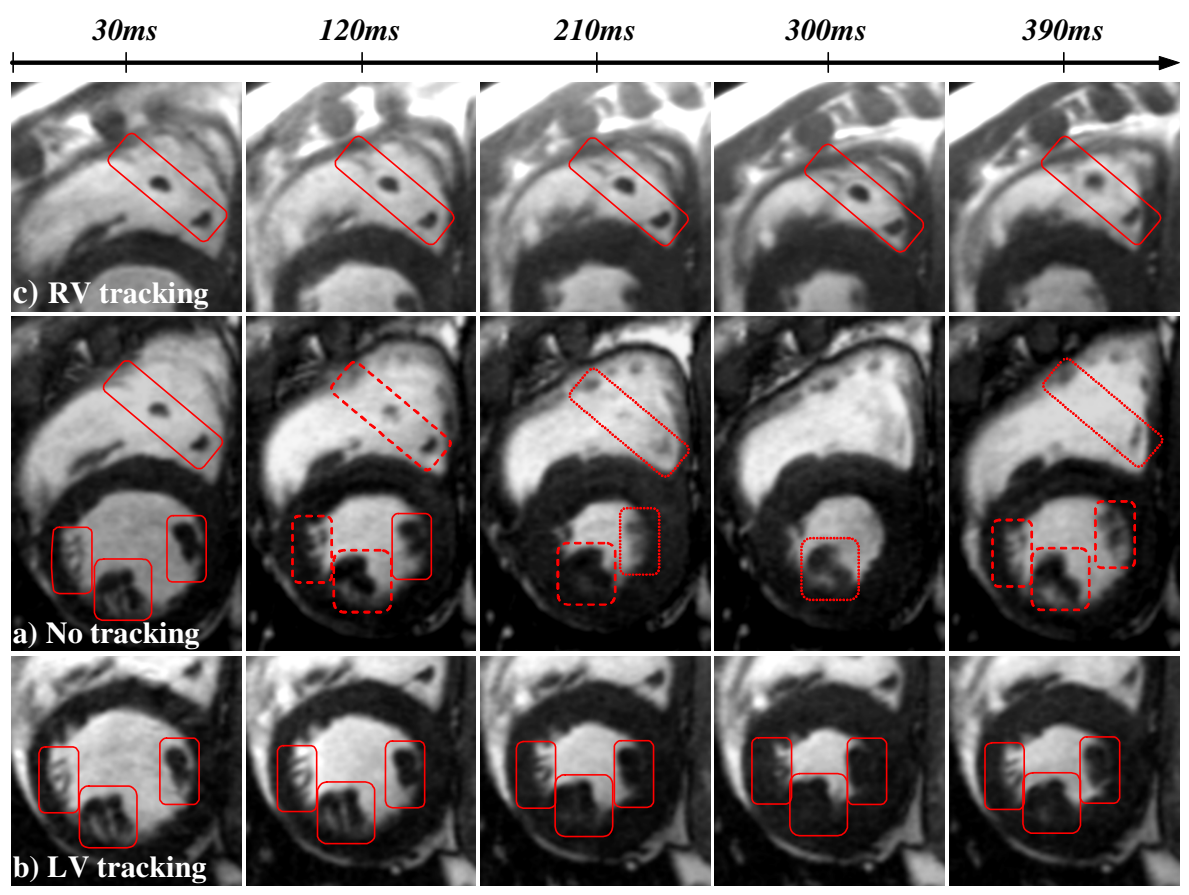

Fig. 2. Static and motion-tracked mid-ventricular short axis acquisitions through systole and early diastole for one of the subjects studied. (a) Conventional MR acquisition with a static imaging plane. The red boxes show localised trabeculae and papillary muscles that then start exhibiting motion blurring (dashed boxes) and begin to move out of the imaging plane (dotted boxes). With adaptive slice-tracking of the (b) left and (c) right ventricles, the trabeculae remain localized throughout the cardiac cycle. 
Table 1. Mean, standard deviation and maximum error of slice position and orientation accuracy between an automatic and a manual tracking $(A v s M)$, and two manual trackings $(M v s$ $M)$, for the left ventricle in 5 subjects and right ventricle (RV) in 1 subject

\begin{tabular}{ccc|cc|cc}
\hline \multirow{2}{*}{$\begin{array}{c}\text { Data- } \\
\text { set }\end{array}$} & Total & Mags & Mis- & \multicolumn{2}{|c|}{ mean/stdev/max position error } & \multicolumn{2}{|c}{ mean/stdev/max orientation error } \\
\cline { 4 - 7 } & Track $M(\mathrm{~mm})$ & $M v s M(\mathrm{~mm})$ & $A v s M\left(^{\circ}\right)$ & $M v s M\left({ }^{\circ}\right)$ \\
\hline 1 & 88 & 2 & $0.68 / 0.37 / 2.28$ & $0.94 / 0.60 / 3.10$ & $1.38 / 0.77 / 4.08$ & $1.45 / 0.78 / 3.62$ \\
2 & 96 & 0 & $1.03 / 0.44 / 2.13$ & $0.86 / 0.35 / 1.74$ & $1.39 / 0.56 / 3.41$ & $1.50 / 0.79 / 3.69$ \\
3 & 88 & 1 & $0.58 / 0.26 / 1.38$ & $0.63 / 0.31 / 1.56$ & $1.30 / 0.83 / 3.44$ & $1.66 / 0.85 / 3.85$ \\
4 & 80 & 0 & $0.93 / 0.45 / 3.23$ & $0.94 / 0.52 / 2.80$ & $1.85 / 0.96 / 4.95$ & $1.88 / 0.94 / 4.56$ \\
$5 \mathrm{LV}$ & 88 & 3 & $0.93 / 0.45 / 2.74$ & $0.88 / 0.72 / 2.95$ & $1.33 / 0.74 / 3.82$ & $1.80 / 0.88 / 3.82$ \\
$5 \mathrm{RV}$ & 88 & 11 & $1.78 / 1.34 / 8.94$ & $2.22 / 1.77 / 8.27$ & $2.73 / 1.66 / 6.95$ & $2.78 / 1.99 / 8.48$ \\
\hline
\end{tabular}

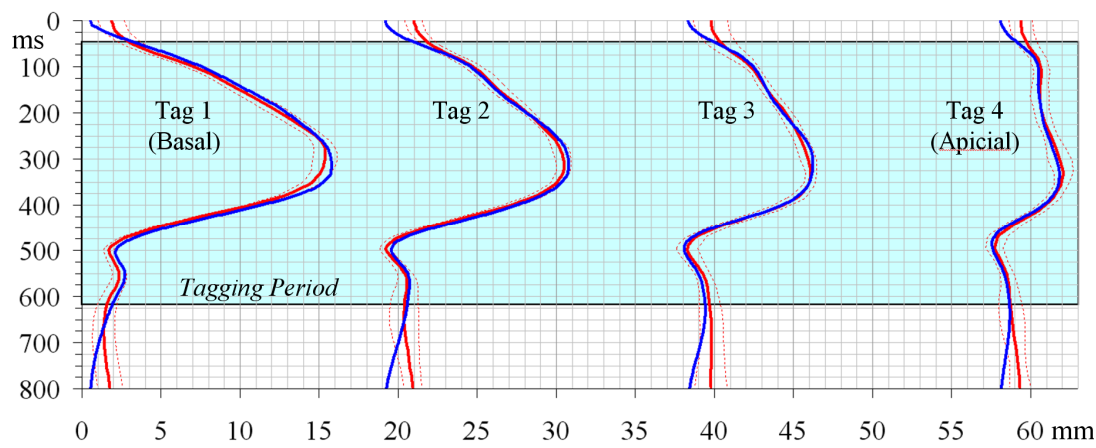

Fig. 3. Through-plane component of the centre of 4 moving slices through the cardiac cycle, derived from 4 left ventricular COMB tags imaged during the period shaded in turquoise. Two standard deviations from the mean of 10 expert manual delineations are shown in red. The automatic approach, including motion-correction for the known tag geometry immediately after the $\mathrm{R}$-wave, is shown in blue.

For motion tracking, since the subject must remain static in the scanner during processing, real-time execution is necessary; the automatic approach takes 30 seconds including verification, as compared mean manual processing of 309 seconds. Validation of the tracking on the 6 datasets is presented in Table 1 and Fig. 3. Table 1 shows that slice position and orientation errors between the proposed automatic tracking and an expert manual delineation are equivalent. The right ventricle was more difficult to track due to the thinner wall and tagged blood remaining in the field of view. The relationship was then analysed further on one left ventricle dataset by obtaining manual delineations from 10 experts, and comparing the normal distribution (confirmed by Shapiro-Wilk tests) of position and orientation motion against the automatic approach. Fig. 3 illustrates tracked long-axis motion of the 4 tags. The automatic approach remained within the $95.5 \%$ confidence interval of the manual approach in $98.2 \%$ of samples. Variance in manual delineation increases at early systole and end diastole due to the issues of tagged blood and tag fade respectively. Furthermore, since the tags are only imaged after $40 \mathrm{~ms}$ due to initialisation of the COMB pre-pulse, 
and before $620 \mathrm{~ms}$ due to tag fade, motion interpolation is required for the other phases. It can be seen in the automatic approach that incorporating the known starting tag location at $2.5 \mathrm{~ms}$ significantly aids tracking outside the tagging period.

An example cylindrical density projection with frozen bulk motion is shown in Fig. 4(b). It can be seen that from $420 \mathrm{~ms}$ onwards, the papillary muscles rotates and shortens relative to the ventricle, as previously observed by invasive means [3]. Circumferential and radial components of the strain (Fig 4(b-c)) were then quantified with the proposed method and the derived strain maps reprojected onto the original volume (Fig. 4(a)). As expected, the radial strain of the myocardium can be seen compressing during systole and expanding during diastole. The circumferential strain is less deterministic, but more pronounced during diastolic relaxation of the muscle, as the trabeculations appear to swirl due to vortices in the filling blood pool. Note that the strain accuracy is affected by the partial volume effect caused by the $6 \mathrm{~mm}$ slice thickness. This issue can be resolved through the use of super-resolution, but will require extension of our previous work [6] since the motion-tracked slices are no longer coplanar.

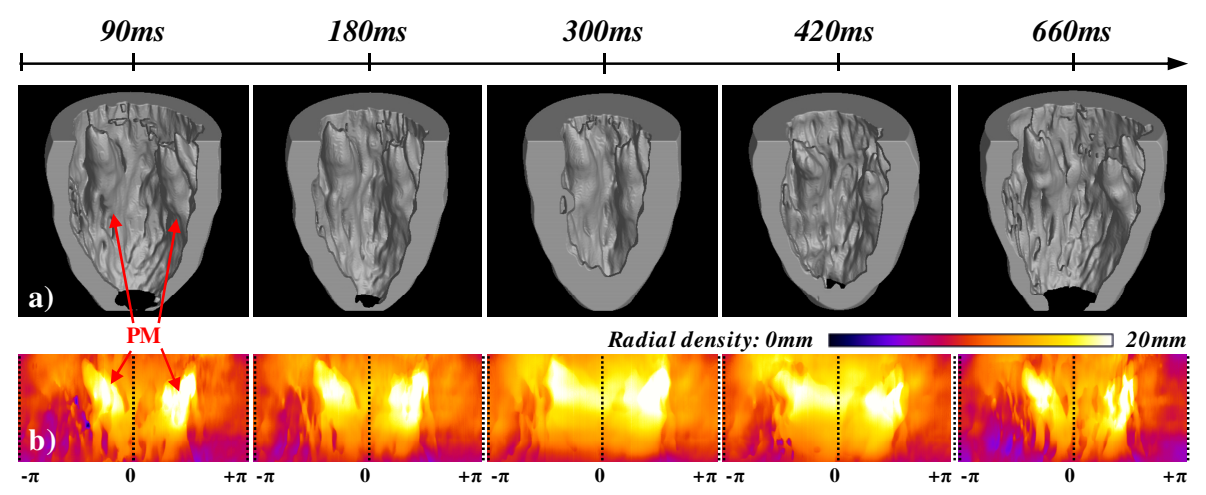

(c) Circumferential and (d) radial relative strain over 30ms: $50 \% \square 200 \%$

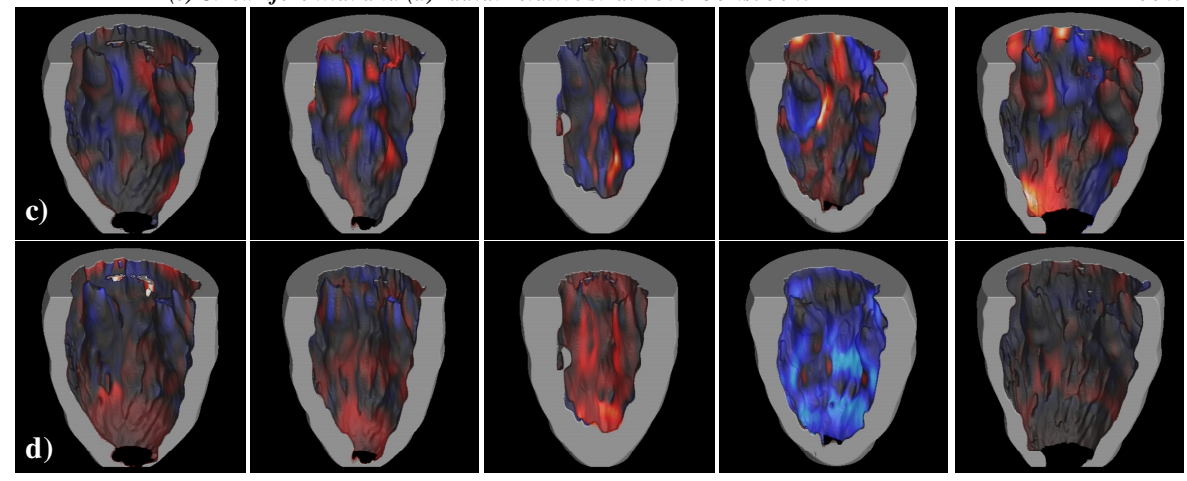

Fig. 4. Views of the left ventricle through the cardiac cycle with frozen through-plane and inplane motion. (a) Volume rendering showing detailed trabeculations and papillary muscles (PM). (b) Unwrapped cylindrical density projection of the volume, with radial angle as $x$-axis and longitudinal depth as $y$-axis. Derived circumferential (c) and radial (d) relative strain of the myocardium, where $50 \%$ represents compression to half previous size over the last $30 \mathrm{~ms}$, and $200 \%$ is expansion by double. The scale is logarithmic i.e. black represents no strain (100\%). 


\section{Conclusions}

In this paper we have demonstrated a novel motion-tracked prospective imaging technique for revealing detailed ventricular trabeculation throughout the cardiac cycle. This is the first time that the motion of the trabeculae and papillary muscles are visualised and quantified in CMR. The morphologic and functional quantification achieved in this study will enhance our understanding and evaluation of cardiac diseases such as regional ischemia, isolated ventricular non-compaction, arrythmogenic ventricular dysplasia, and mitral insufficiency. Furthermore, the morphological structure revealed in this study will allow CFD analysis for elucidating the haemodynamic role of the trabeculations. The proposed multi-slice adaptive imaging technique will also have significant implications for CMR sequence design, including motioncompensated measurements of flow and myocardial perfusion, which are particularly sensitive to bulk cardiac motion. Furthermore, it is hoped that imaging sequences requiring long acquisition windows $e . g$. for small structures such as the coronary arteries, or T1 relaxation imaging, can now be utilized at any point in the cardiac cycle.

\section{References}

1. Petersen, S.E., Selvanayagram, J.B., Wiesmann, F., Robson, M.D., Francis, J.M., Anderson, R.H., Watkins, H., Neubauer, S.: Left Ventricular Non-Compaction. J. Am. Coll. Card 46, 101-105 (2005)

2. Kiumura, F., Sakai, F., Sakomura, Y., Fujimura, M., Ueno, E., Matsuda, N., Kasanuki, H., Mitsuhasi, N.: Helical CT Features of Arrhythmogenic Right Ventricular Cardiomyopathy. Radiographics 22, 1111-1124 (2002)

3. Levine, R.A., Vlahakes, G.J., Lefebvre, X., Guerrero, L., Cape, E.G., Yoganathan, A.P., Weyman, A.E.: Papillary Muscle Displacement Causes Systolic Anterior Motion of the Mitral Valve. Circulation 91, 1189-1195 (1995)

4. Karwatowski, S.P., Mohiaddin, R., Yang, G.-Z., Firmin, D.N, Sutton, M.S., Underwood, S.R., Longmore, D.B.: Assessment of regional left ventricular long-axis motion with MR velocity mapping in healthy subjects. J. Magn. Reson. Imaging 4, 151-155 (1994)

5. Axel, L.: Papillary Muscles Do Not Attach Directly to the Solid Heart Wall. Circulation 109, 3145-3148 (2004)

6. Dowsey, A.W., Lerotic, M., Keegan, J., Thom, S., Firmin, D., Yang, G.-Z.: MotionCompensated MR Valve Imaging with COMB Tag Tracking and Super-Resolution Enhancement. In: Larsen, R., Nielsen, M., Sporring, J. (eds.) MICCAI 2006. LNCS, vol. 4191, pp. 364-371. Springer, Heidelberg (2006)

7. Tustison, N., Amini, A.A.: Biventricular Myocardial Strains via Non-Rigid Registration of Anatomical NURBS Models. IEEE Trans. Med. Imag. 25, 94-112 (2006)

8. Kozerke, S., Scheidegger, M.B., Pedersen, E.M., Boesiger, P.: Heart motion adapted cine phase-contrast flow measurements through the aortic valve. Magn. Reson. Med. 42, 970978 (1999)

9. Thévenaz, P., Blu, T., Unser, M.: Interpolation Revisited. IEEE Trans. Med. Imag. 19, 739-758 (2000)

10. Dowsey, A.W., English, J., Pennington, K., Cotter, D., Stuehler, K., Marcus, K., Meyer, H.E., Dunn, M.J., Yang, G.-Z.: Examination of 2-DE in the Human Proteome Organisation Brain Proteome Project pilot studies with the new RAIN gel matching technique. Proteomics 6, 5030-5047 (2006)

11. Nocedal, J.: Updating quasi-Newton matrices with limited storage. Math. Comp. 35, 773-782 (1980) 